\title{
"Weekend Effect" on 30-Day Readmissions among Stroke Survivors: An Analysis of the National Readmission Database
}

\author{
Bijan J. Khaksari ${ }^{a, b} \quad$ Erin R. Kulick ${ }^{a}$ b Mitchell S.V. Elkind ${ }^{a}$ b Amelia K. Boehme ${ }^{a, b}$ \\ ${ }^{a}$ Department of Epidemiology, Mailman School of Public Health, Columbia University, \\ New York, NY, USA; ${ }^{b}$ Department of Neurology, Vagelos College of Physicians and Surgeons, \\ Columbia University, New York, NY, USA
}

\section{Keywords}

Ischemic stroke $\cdot$ Stroke quality of care $\cdot$ Weekend admission

\section{Abstract}

Background and Purpose: Previous studies suggested that quality of care may be lower on weekends than during the week. We hypothesized that, among patients hospitalized for an index ischemic stroke, those admitted on weekends would have a higher risk of 30-day readmission than those admitted on weekdays. Methods: We utilized the 2013 Nationwide Readmission Database, which includes data on US inpatient admissions from the Agency for Healthcare Research and Quality Healthcare Utilization Project. The database includes a nationally representative weighted probability sample of inpatient hospitalizations regardless of insurance status. Patients with primary acute ischemic stroke were identified using previously validated ICD-9$\mathrm{CM}$ diagnosis codes. We conducted a weighted analysis using survey design logistic regression models to estimate crude and adjusted odds ratios (OR) and $95 \%$ confidence intervals $(95 \% \mathrm{Cl}$ ) for the association between weekend admission and 30-day readmission in ischemic stroke patients. Results: Among 319,317 patients admitted for ischemic stroke, 12.1\% were readmitted within 30 days. Those with 30-day readmissions had an average of 8 chronic conditions, and all cardiovascular-related comorbidities increased the risk of 30 -day readmissions. Ischemic stroke patients admitted on weekends had odds of 30-day readmission similar to patients admitted on weekdays (OR 1.02; 95\% Cl 0.98-1.06). Weekend admission also did not affect readmission at 7 or 60 days. Conclusions: We found no association between weekend admission and 30day readmissions, providing indirect evidence of homogeneity in the quality of care delivered during week day and weekend admissions. 


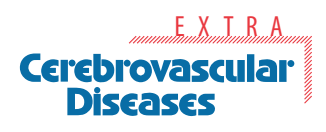

\begin{tabular}{l|l}
\hline Cerebrovasc Dis Extra 2019;9:66-71 \\
\hline DOI: 10.1159/000500611 & $\begin{array}{l}\text { (c) 2019 The Author(s). Published by S. Karger AG, Basel } \\
\text { www.karger.com/cee }\end{array}$ \\
\hline
\end{tabular}

Khaksari et al.: Weekend Admissions and 30-Day Readmissions for Stroke: An Analysis of the NRD

\section{Introduction}

Hospitals and payers increasingly utilize 30-day readmission (30dRA) as a quality-ofcare and cost reduction metric [1]. Readmission within a short time period after a stroke increases morbidity and mortality. Recent work showed that $12.1 \%$ of acute ischemic stroke (AIS) patients were readmitted within 30 days; $89.6 \%$ of the readmissions were considered unplanned and $12.9 \%$ were preventable [2].

Hospital services and quality of care that impact 30dRA may differ between weekends and weekdays. The discrepancy in discharge outcomes related to weekend versus weekday admission, termed the "weekend effect," has been associated with adverse health outcomes for 23 of the 100 leading causes of death [3]. Previous studies indicated that weekend stroke admissions had a higher 30-day case fatality rate than admissions on week days [4-6]. A more recent study has shown similar 30-day case-fatality rates between week day and weekend admissions, but it was conducted in comprehensive stroke centers [7]. This may not be generalizable as dedicated stroke units that comply with comprehensive stroke unit principles may be protective against hospital case fatality due to the requirements needed for comprehensive stroke center certification [8]. Furthermore, the effect of weekend admission on 30dRA after stroke has not been assessed.

We hypothesized that stroke patients admitted at weekends would have higher odds of 30dRA than patients admitted on week days (i.e., Monday to Friday).

\section{Methods}

\section{Study Population}

We utilized the Agency for Healthcare Research and Quality (AHRQ) Healthcare Utilization Project Nationwide Readmission Database (NRD) for 2013, a nationally representative, weighted probability sample of inpatient hospitalizations in the USA, regardless of insurance status. International Classification of Diseases Ninth Revision (ICD-9) codes (433.x1, 434.x1, and 436) were used to identify patients admitted with AIS. An AIS code in the first diagnostic position was classified as a primary stroke code. All patients with a primary AIS diagnosis of stroke were identified, and their initial (index) hospital admission was tagged for further investigation.

\section{Exposure, Outcome, and Covariate Definitions}

The exposure of interest, admission during a weekend, is a variable collected and reported in the NRD. The NRD defined weekend admissions as admission from 12:01 a.m. Saturday to 11:59 p.m. Sunday [9]. Given that the data in our analysis covered a 1-year time span, we stopped our analysis on 1 December, to ensure an entire 30-day period for a patient's readmission to occur.

We defined 30dRA as any admission within 30 days of index hospitalization discharge. We excluded same-day events, readmissions exceeding 30 days from index hospitalization discharge, and in-hospital deaths. In a sensitivity analysis, we examined whether admission on the weekend was associated with 7-day and 60-day readmissions of AIS patients. Using previously validated methods, we classified readmission events as planned or unplanned, also identifying the number of readmissions deemed preventable due to ambulatory care-sensitive conditions [10]. Demographic characteristics such as age, sex, insurance type, number of chronic conditions, hospital teaching status, hospital bed size, and length of stay are saved in the NRD. Treatments including intravenous tissue-type plasminogen activator (tPA; ICD-9 code 99.1) or endovascular therapy (ICD-9 code 39.74) were determined by using procedure codes. AHRQ comorbidity measures using ICD-9-CM codes were assessed as potential covariates. 
Khaksari et al.: Weekend Admissions and 30-Day Readmissions for Stroke: An Analysis of the NRD

Table 1. Baseline demographics for patients readmitted or not readmitted within 30 days of hospitalization for stroke

\begin{tabular}{|c|c|c|c|}
\hline & $\begin{array}{l}\text { Total } \\
(n=319,317)\end{array}$ & $\begin{array}{l}\text { Readmitted } \\
\text { within } 30 \text { days } \\
(n=38,625)\end{array}$ & $\begin{array}{l}\text { Not readmitted } \\
\text { within } 30 \text { days } \\
(n=280,692)\end{array}$ \\
\hline Age, years & $70.7 \pm 0.12$ & $71.8 \pm 0.14$ & $70.6 \pm 0.13$ \\
\hline Female & 51.6 & 51 & 51.6 \\
\hline \multicolumn{4}{|l|}{ Medical insurance } \\
\hline Medicare & 67.9 & 73.2 & 67.1 \\
\hline Medicaid & 6.9 & 7.6 & 6.8 \\
\hline Private & 16.9 & 13.3 & 17.3 \\
\hline Other & 5.1 & 3.3 & 5.3 \\
\hline \multicolumn{4}{|l|}{ Hospital bed size } \\
\hline Small & 10.6 & 10.1 & 10.7 \\
\hline Medium & 23.9 & 23.7 & 24.0 \\
\hline Large & 65.5 & 66.2 & 65.3 \\
\hline \multicolumn{4}{|l|}{ Hospital teaching status } \\
\hline Metropolitan nonteaching & 38.9 & 39.9 & 38.8 \\
\hline Metropolitan teaching & 49.4 & 49.5 & 49.4 \\
\hline Nonmetropolitan & 11.7 & 10.6 & 11.9 \\
\hline \multicolumn{4}{|l|}{ Hospital RUCA code } \\
\hline Urban & 51.4 & 53.9 & 51.0 \\
\hline Large rural town & 36.9 & 35.4 & 37.1 \\
\hline Small rural town & 8.7 & 7.9 & 8.8 \\
\hline Isolated & 2.9 & 2.6 & 3.1 \\
\hline Number of chronic conditions & $7.1 \pm 0.04$ & $7.9 \pm 0.05$ & $7.0 \pm 0.04$ \\
\hline Hypercoagulable & 3.1 & 4.1 & 2.9 \\
\hline Hypertension & 77.7 & 76.4 & 77.9 \\
\hline $\mathrm{CHF}$ & 5.1 & 7.4 & 4.8 \\
\hline Chronic ischemic heart disease & 24.4 & 29.4 & 23.7 \\
\hline Pulmonary hypertension & 2.6 & 3.5 & 2.5 \\
\hline Valvular heart disease & 6.8 & 7.6 & 6.7 \\
\hline Renal failure & 18.2 & 25.2 & 17.2 \\
\hline Diabetes & 35.7 & 38.8 & 35.3 \\
\hline Weekend admission & 25.5 & 26.1 & 25.5 \\
\hline tPA use & 6.7 & 6.5 & 6.8 \\
\hline IAT final & 0.9 & 1.1 & 0.9 \\
\hline \multicolumn{4}{|l|}{ APDRG severity } \\
\hline Minor loss of function & 15.6 & 19.5 & 16.4 \\
\hline Moderate loss of function & 51.5 & 45.1 & 52.3 \\
\hline Major loss of function & 27.4 & 35.9 & 26.2 \\
\hline Extreme loss of function & 5.6 & 9.5 & 5.0 \\
\hline Length of stay, days & $4.8 \pm 0.04$ & $6.1 \pm 0.09$ & $4.6 \pm 0.04$ \\
\hline
\end{tabular}

Values are expressed as percentages or mean \pm SD. RUCA, rural-urban commuting area; CHF, congestive heart failure; APDRG, all patient refined diagnosis related groups; tPA, tissue-type plasminogen activator; IAT, intra-arterial thrombolytic therapy.

\section{Statistical Analysis}

We computed proportions of patients with 30-day readmission overall, and unplanned and potentially preventable readmissions. Survey design logistic regression models were fit to estimate weighted crude odds ratios (OR) and 95\% confidence intervals (CI) for the association between weekend admission and 30dRA. Considering the results were not significant, we did not adjust the models for any covariates of interest. 
Table 2. Unadjusted association of weekend admission for acute ischemic stroke and readmissions

\begin{tabular}{lllll}
\hline & $\begin{array}{l}\text { Preventable } \\
\text { 30-day RA }\end{array}$ & $\begin{array}{l}\text { All-cause } \\
\text { 30-day RA }\end{array}$ & $\begin{array}{l}\text { All-cause } \\
\text { 7-day RA }\end{array}$ & $\begin{array}{l}\text { All-cause } \\
\text { 60-day RA }\end{array}$ \\
\hline Weekend admission & $1.03(0.99-1.08)$ & $1.02(0.98-1.06)$ & $1.01(0.95-1.07)$ & $1.01(0.98-1.05)$ \\
\hline
\end{tabular}

Values are expressed as odds ratio (95\% confidence interval). dRA, readmission.

\section{Results}

Of 319,317 patients with an index AIS, 38,625 (12.1\%) experienced 30dRA. Patient demographics stratified by $30 \mathrm{dRA}$ are shown in Table 1 . Approximately $26 \%$ of readmitted patients were initially a weekend admission. Age did not differ between those with and without $30 \mathrm{dRA}$ (71.8 vs. 70.6 years). Patients who experienced 30dRA also had more chronic conditions (mean 7.90.1) than those without 30dRA (mean 7.00.05). Index AIS patients with 30dRA also had a longer stay, increased All Patient Refined DRG severity (APDRG Severity), more cardiovascular-related comorbid conditions, less treatment with tPA, and higher mortality.

There was no association between weekend admission and all-cause 30dRA (OR 1.02; 95\% CI 0.98-1.06) or between weekend admission and potentially preventable 30dRA (OR $1.03,95 \%$ CI 0.99-1.08). There was no association of weekend admission on 7dRA (OR 1.01, 95\% CI 0.95-1.07) or 60dRA (OR 1.01, 95\% CI 0.98-1.05) (Table 2).

The final models were not adjusted for Table 1 elements due to no association between our primary predictor and primary outcome. Overall, the patients who were readmitted had a higher proportion of poor outcomes after their readmission hospitalization than the stroke patients who were not readmitted.

\section{Discussion}

We found no relationship between weekend admissions and 30-day readmissions among AIS patients, indicating that the "weekend effect" previously described for other acute conditions may not extend to all post-stroke outcomes. Neither all-cause nor potentially preventable readmissions were associated with a weekend admission.

Our results were consistent with some other US studies suggesting that weekend AIS admissions have similar quality-of-care outcomes to those for week days [7, 11]. However, 30-day case fatality was the primary outcome of interest in those papers whereas we utilized 30-day readmissions in our study. The suggested decrease in stroke-related fatality indicates that other outcomes such as readmission should be investigated when exploring potential differences in quality of care. The absence of a weekend effect in our study could be due to an improved quality of care compared to in studies conducted previously. The discrepancy in discharge outcomes, particularly case fatality, related to weekend versus week day admissions, has been associated with adverse health outcomes for 23 of the 100 leading causes of death [3]. Studies investigating the weekend effect on mortality during admission around the world, e.g., in England, Australia, and Taiwan, show an increased risk of mortality from acute medical conditions such as myocardial infarctions during weekend admissions [12-14]. Hospital access and homogeneity of 24-h coverage 7 days a week may be influential factors to improved quality of care. Similar proportions of senior staffing 7 days a week who are capable of performing elective procedures, such as tPA, can potentially decrease adverse 
Khaksari et al.: Weekend Admissions and 30-Day Readmissions for Stroke: An Analysis of the NRD

ischemic stroke outcomes. A reduced length of stay, a decreased risk of nosocomial infection, advanced neuroimaging, and early outpatient follow-up are factors that may have additionally improved quality of care over time. Other factors, such as whether the hospital is an academic center, or has a dedicated stroke unit, might influence post-stroke outcomes including readmission [7]. We explored whether these associations differed in different subgroups, but they remained the same in the subgroup analyses.

Our study is not without limitations. The NRD is an administrative dataset, and the ICD-9 CM codes are primarily used for billing purposes with a limited number of data elements. Since this study is based in the USA, the results may not extend to other health systems or countries. We had no information on severity of stroke, stroke etiology, stroke subtype, or individual functional ability prior to stroke, which could influence both weekend admissions and 30-day readmission after a stroke. Future studies should use either the US National Institutes of Health Stroke Score or the level of consciousness as a surrogate for stroke severity. We had no information on whether patients died after leaving the hospital, and no information on health care utilization patterns that might prevent readmission. Finally, these results are only generalizable to ischemic stroke hospital readmissions that take place in the USA.

These results demonstrate that 30-day readmissions due to ischemic stroke hospitalizations during the business week and at the weekend are similar. We inspected 7-day, 30-day, and 60-day readmissions to capture critical time intervals that would potentially allude to specific quality-of-care predictors. We used a large, nationally representative dataset that meant that a false-negative result was unlikely. While optimistic, these results require confirmation in future prospective studies.

\section{Statement of Ethics}

The Institutional Review Board at our center waived review of this study due to the absence of identifiable patient data.

\section{Disclosure Statement}

The authors declare that there are no conflicts of interest to report. The content is solely the responsibility of the authors and does not necessarily represent the official views of the NINDS or the NIH.

\section{Funding Sources}

Dr. Boehme is supported by NINDS NIH R03 NS101417 and NINDS NIMHD R21 MD012451. Dr. Elkind serves on the National, Founders Affiliate, and New York City chapter boards of the American Heart Association/American Stroke Association, and receives royalties from UpToDate for chapters related to cryptogenic stroke.

\section{References}

1 Condon C, Lycan S, Duncan P, Bushnell C. Reducing Readmissions after Stroke with a Structured Nurse Practitioner/Registered Nurse Transitional Stroke Program. Stroke. 2016; Jun;47(6):1599-604.

2 Vahidy FS, Donnelly JP, McCullough LD, Tyson JE, Miller CC, Boehme AK, et al. Nationwide Estimates of 30-Day Readmission in Patients with Ischemic Stroke. Stroke. 2017 May;48(5):1386-8.

3 Bell CM, Redelmeier DA. Mortality among patients admitted to hospitals on weekends as compared with weekdays. N Engl J Med. 2001 Aug;345(9):663-8.

4 Sorita A, Ahmed A, Starr SR, Thompson KM, Reed DA, Dabrh AM, et al. Off-hour presentation and outcomes in patients with acute ischemic stroke: a systematic review and meta-analysis. Eur J Intern Med. 2014 Apr;25(4): 394-400.

5 Saposnik G, Baibergenova A, Bayer N, Hachinski V. Weekends: a dangerous time for having a stroke? Stroke. 2007 Apr;38(4):1211-5. 
Khaksari et al.: Weekend Admissions and 30-Day Readmissions for Stroke: An Analysis of the NRD

6 Sharp AL, Choi H, Hayward RA. Don't get sick on the weekend: an evaluation of the weekend effect on mortality for patients visiting US EDs. Am J Emerg Med. 2013 May;31(5):835-7.

7 Albright KC, Raman R, Ernstrom K, Hallevi H, Martin-Schild S, Meyer BC, et al. Can comprehensive stroke centers erase the 'weekend effect'? Cerebrovasc Dis. 2009;27(2):107-13.

8 Bekelis K, Marth NJ, Wong K, Zhou W, Birkmeyer JD, Skinner J. Primary Stroke Center Hospitalization for Elderly Patients with Stroke: Implications for Case Fatality and Travel Times. JAMA Intern Med. 2016 Sep; 176(9):1361-8.

9 Magid D, Wang Y, Mcnamara RL, et al. Relationship Between Time of Day, Day of Week, Timeliness of Reperfusion, and In-Hospital Mortality for Patients Myocardial Infarction. JAMA. 2005;294(7):803-12.

10 Campbell JT, Bray BD, Hoffman AM, Kavanagh SJ, Rudd AG, Tyrrell PJ; Intercollegiate Stroke Working Party. The effect of out of hours presentation with acute stroke on processes of care and outcomes: analysis of data from the Stroke Improvement National Audit Programme (SINAP). PLoS One. 2014 Feb;9(2):e87946.

11 Hoh BL, Chi YY, Waters MF, Mocco J, Barker FG 2nd. Effect of weekend compared with weekday stroke admission on thrombolytic use, in-hospital mortality, discharge disposition, hospital charges, and length of stay in the Nationwide Inpatient Sample Database, 2002 to 2007. Stroke. 2010 Oct;41(10):2323-8.

12 Aldridge C, Bion J, Boyal A, Chen YF, Clancy M, Evans T, et al; HiSLAC Collaborative. Weekend specialist intensity and admission mortality in acute hospital trusts in England: a cross-sectional study. Lancet. 2016 Jul; 388(10040):178-86.

13 Clarke MS, Wills RA, Bowman RV, Zimmerman PV, Fong KM, Coory MD, et al. Exploratory study of the 'weekend effect' for acute medical admissions to public hospitals in Queensland, Australia. Intern Med J. 2010 Nov; 40(11):777-83.

14 Hsieh CY, Lin HJ, Chen CH, Li CY, Chiu MJ, Sung SF. “Weekend effect” on stroke mortality revisited: application of a claims-based stroke severity index in a population-based cohort study. Medicine (Baltimore). 2016 Jun; 95(25):e4046. 